\title{
Shoulder muscle weakness effects on muscle hardness around the shoulder joint and scapula
}

\section{Kubota Atsushi*, Takayanagi Chiho and Kishimoto Kohei}

Department of Sports Medicine, Juntendo University, Chiba, Japan

\section{Abstract}

Purpose: The time course of muscle stiffness of muscles around the shoulder joint and the scapula was investigated according to the degree of muscle weakness. This study was conducted to clarify the recovery process of muscle hardness of the muscles surrounding the shoulder joint and the scapula after the shoulder internal and external rotational exercises.

Methods: Participants were 7 healthy men $(23.6 \pm 1.4 \mathrm{yr})$, repeated internal and external rotations of the shoulder joint until the mean work of three internal and external rotations each was less than $90 \%, 80 \%$, or $70 \%$ of the standard. Muscle hardness of the supraspinatus muscle, the infraspinatus muscle, and the rhomboideus muscle was measured before, immediately after, and 1 to $72 \mathrm{hr}$ after each bout of exercise.

Muscle hardness was measured as Strain ratio using an ultrasound real-time tissue elastography. In addition, the rates of change were calculated using muscle hardness before exercise as the standard, to compare differences in the rate of change after exercise between conditions.

Results: The rates of change of the Strain ratio between measurements taken before and after exercise were compared among conditions for the infraspinatus muscle. Results were $-7.1 \pm$ $5.3,-15.2 \pm 10.3$, and $-25.0 \pm 8.8$, respectively, at $90 \%, 80 \%$, and $70 \%$, with a significant difference between a decrease to $90 \%$ and to $70 \%(p<0.05)$. Significant difference was found in the change over time for the infraspinatus muscle only between values obtained immediately after exercise and after $72 \mathrm{hr}$ at a decrease to $70 \%(p<0.05)$.

Conclusion: Those results described above demonstrated that the infraspinatus muscle and the supraspinatus muscle were harder immediately after exercise when the shoulder joint was at a higher degree of muscle weakness, and demonstrated that the change was likely to be recovered after $72 \mathrm{hr}$.

\section{More Information}

*Address for Correspondence: Kubota Atsushi, Department of Sports Medicine, Juntendo University, Chiba, 1-1 Hiragagakuendai, Inzai, Chiba, 270-1695, Japan, Tel: +81-476-98-1001; FAX: +81-476-98-1011

Email: akubota@juntendo.ac.jp

Submitted: 14 April 2020

Approved: 20 April 2020

Published: 21 April 2020

How to cite this article: Atsushi $\mathrm{K}$, Chiho $\mathrm{T}$, Kohei K. Shoulder muscle weakness effects on muscle hardness around the shoulder joint and scapula. J Sports Med Ther. 2020; 5: 001-007.

DOI: 10.29328/journal.jsmt.1001049

Copyright: (c) 2020 Atsushi $\mathrm{K}$, et al. This is an open access article distributed under the Creative Commons Attribution License, which permits unrestricted use, distribution, and reproduction in any medium, provided the original work is properly cited.

Keyword: Baseball shoulder; Elastography; Internal and external rotation; Conditioning

Check for updates

OPEN ACCESS

\section{Background}

Sports associated with overhead motions with extremely different frequencies of use of the right and left arms are likely to cause notable differences in morphology and functions between right and left arms. An example of such a sport is baseball, particularly pitching. Pitchers throw a ball more frequently than players at the other positions. Moreover, they are presumed to be more affected by pitching [1]. Surveys of the shoulder joint [2-4] and the elbow joint [5] in baseball players of various skill levels and ages have been conducted. Some reports particularly describe that they have a smaller range of motion for internal rotation in the shoulder joint of the pitching arm. This range of motion is regarded as a difficulty that increases the risk of injury to the shoulder and the elbow [6-8]. Reportedly, muscles and articular capsules located posterior to the shoulder joint of the pitching arm are stiffer than those of the non-pitching arm, which is regarded as a factor decreasing the range of motion for internal rotation in the shoulder joint. It increases risk of injury to the shoulder $[9,10]$. Earlier reports have described that it is not the excessive decrease in the degree of internal rotation in the shoulder joint that increases the risk of injury to the shoulder and elbow of a pitcher: the risk is posed by hypofunction in the supraspinatus muscle before the baseball season $[8,11]$. Moreover, muscle weakness not only of the supraspinatus muscle but also of the shoulder external rotator muscles is regarded as an 
important difficulty that may induce the shoulder and elbow problems. Earlier reports show that they might induce upper limb disorder because of the overhead motions used during pitching [12-14]. As described above, not only functions such as the range of motion and muscle strength of the shoulder joint, but the state of muscles around the joint are important to prevent pitching injuries. However, the relation between joint functions and the state of surrounding muscles has not been fully investigated.

Although increased muscle stiffness around the shoulder joint is regarded as one of the factors inducing pitching injuries, earlier reports have described that static stretching of internal rotation in the shoulder joint decreases muscle stiffness in the infraspinatus muscle [15]. The range of motion of the shoulder joint might be improved. Injuries and disorders in athletes with repeated overhead motions might be prevented by decreasing stiffness in the rotator cuff, which comprises the subscapular muscle, the supraspinatus muscle, the infraspinatus muscle, and the teres minor muscle [9]. These results indicate that the conditioning of these four muscles is indispensable for the prevention of pitching injuries in baseball. Nevertheless, it remain unknown how the strength or amount of pitching affects muscles that comprise the rotator cuff. It is therefore difficult to condition the rotator cuff in pitching properly. A need exists to investigate how the functions and states of muscles comprising the rotator cuff change by actually adjusting the strength and amount of pitching, but a risk of injury caused by repeated pitching exists until hypofunction occurs. In this survey, we induced only hypofunction around the shoulder joint by means other than pitching. The hypofunction was set stepwise based on the degree of muscle weakness. To clarify the recovery process, the time course of muscle hardness of muscles around the shoulder joint and the scapula was investigated by the degree of muscle weakness. The purpose of this study was to clarify the recovery process of muscle hardness of the muscles surrounding the shoulder joint and the scapula after shoulder internal and external rotational exercises. We hypothesized that the muscle hardness increases according to the degree of muscle weakness induced by the shoulder internal and external rotational exercises, and it takes longer time for the muscle hardness to recover to the normal value when the degree of muscle weakness is larger.

\section{Methods}

Participants were 7 healthy adult men $(23.6 \pm 1.4$ years of age, $172.6 \pm 2.5 \mathrm{~cm}$ height, and $67.1 \pm 3.2 \mathrm{~kg}$ weight) with no injury or disorder around the shoulder joint. This study was conducted in accordance with the Declaration of Helsinki and was approved by the research ethics committee of Juntendo University Faculty of Health and Sports Science (Jundai-Su-Rin No. 27-2). Informed consent was obtained from all participants after providing participants appropriate oral and written explanations on the study objective, details, procedures, foreseeable risks and measures and compensations before the experiment.

The experimental procedure in this study was the following: The participants first spent 3 days ( $72 \mathrm{hr}$ ) of daily life as usual with restriction on vigorous exercise. Muscle hardness was measured around the right shoulder joint and the scapula of the participants, with subsequent measurements at $1,3,5$, 24, 48 and $72 \mathrm{hr}$ after the initial measurement. The resulting data were used to clarify the change in muscle hardness that might occur in the daily life of the participant. Then the participants sat with the shoulder joint at 90 deg of abduction, and repeated internal and external rotations of the shoulder joint in concentric contraction at an angular velocity of 120 deg/s (BIODEX system4; BIODEX, NY) until predetermined hypofunction was apparent. The hypofunction was calculated using the work of the first internal and external rotation at each time of exercise as the standard. The exercise was repeated until the mean work of 3 internal and external rotations each was less than $90 \%, 80 \%$, or $70 \%$ of the standard. All participants exercised under these three conditions, making sure to confirm that muscle hardness around the shoulder joint was recovered to the initial state and confirming a rest period for a few days before the next condition. Muscle hardness was measured before, immediately after, and 1, 3, 5, 24, 48 and $72 \mathrm{hr}$ after each exercise bout.

Muscle hardness was measured using real-time tissue elastography (RTE) installed in the digital ultrasonic diagnostic equipment (Noblus; Hitachi Ltd., Tokyo). The stiffness reference material was loaded on the linear probe of this device using a dedicated attachment between the site of measurement and the probe, to fit the major axis along the direction of all muscles. The measurement site was mildly compressed with the probe manually and rhythmically. The rate and strength of compression was adjusted to be constant referring to the graph indicated on the display of the equipment. The strain ratio (SR) was calculated with the SR function integrated in the measuring instrument using the clearest image obtained during the compression procedure, where the SR function is a function to calculate the strain ratio between two areas selected in the RTE image of the equipment. The target area was set with the stiffness reference material as the reference area (A), and the participant muscle as the participant area (B), to divide the participant area by the reference area (B/A). This ratio was used as SR in this study to evaluate muscle hardness semiquantitatively. SR is smaller when the muscle is stiffer.

Muscle hardness was measured in three muscles: the supraspinatus muscle, the infraspinatus muscle, and the rhomboideus muscle. Measurement for the supraspinatus muscle was performed in the sitting position with the shoulder joint in slight internal rotation, and for the infraspinatus muscle and the rhomboideus muscle in the abdominal position with the shoulder joint at $90 \mathrm{deg}$ of abduction. The participants were instructed to relax in all measurements. For 
the supraspinatus muscle, the probe tip was placed on the intersection of the medial border of scapula and the scapular spine. The probe was slid upward while checking on the display to ascertain the point at which the scapular spine disappeared. For the infraspinatus muscle, the probe tip was placed on the intersection of the medial border of scapula and the scapular spine. The probe was slid downward while checking on the display to ascertain the point at which the scapular spine disappeared. For the rhomboideus muscle, the middle point of a line between the spinous process of fourth thoracic vertebra and the inferior angle of scapula was marked, where the probe was placed to measure. Measurements were taken in triplicate at each measurement site. The mean of triplicate measurements was used for analyses. The measurement site was marked with a permanent marker at the time of the first measurement to ensure that all subsequent measurements were taken at the same position.

\section{Statistical processing and evaluation method}

The obtained values were checked for the normality using the Leven's test before undergoing the statistical processes. Paired $t$-tests were used to compare differences in SR obtained before and immediately after exercise under respective hypofunction conditions for all muscles. The rate of change between them (SR immediately after exercise/ SR before exercise) was calculated for comparison between conditions using one-way repeated measures analysis of variance (ANOVA) and the Bonferroni method. In addition, the rates of change were calculated respectively at $1,3,5$, 24, 48, after $72 \mathrm{hr}$ using SR before exercise as the standard, to compare differences in the rate of change over time after exercise between conditions by one-way repeated measures ANOVA and Bonferroni method. Additionally, significance was inferred for results with a statistic $<5 \%$.

Furthermore, the standard deviation (SD) was calculated as usual based on SR obtained on three days of daily life. The range of $\pm 2 \mathrm{SD}$ is the $95 \%$ confidence interval. Therefore any change within this range was regarded as possible in daily life. Deviations from the range of the mean \pm 2 SD before exercise were evaluated as clear changes attributable to exercise in this study. All statistical procedures were completed using SPSS ver. 22.0 (IBM Corp. Armonk, NY, USA).

\section{Results}

Comparison of results obtained between before and after exercise showed significant differences in two conditions of the supraspinatus muscle, which was $0.78 \pm 0.48$ before exercise and $0.66 \pm 0.49$ immediately after exercise at a decrease to $80 \%$, and $0.66 \pm 0.39$ before exercise and $0.49 \pm 0.41$ immediately after exercise at $70 \%(p<0.05)$. The infraspinatus muscle was $2.38 \pm 0.94$ before exercise and 2.23 \pm 0.96 immediately after exercise at a decrease to $90 \%(p<$ 0.01 ) $2.35 \pm 0.91$ before exercise and $2.01 \pm 0.89$ immediately after exercise at $80 \%(p<0.05)$, and $2.23 \pm 0.42$ before exercise and $1.67 \pm 0.38$ immediately after exercise at $70 \%(p<0.01)$. Significant differences were found for all the conditions. The rhomboideus muscle showed no significant difference before and after exercise in any condition. The rate of change (immediately after exercise/ before exercise) was compared between conditions for each muscle. No significant difference was found for the supraspinatus muscle or the rhomboideus muscle, although they were $-7.1 \pm 5.3,-15.2 \pm 10.3$, and $-25.0 \pm$ 8.8 , respectively, at $90 \%, 80 \%$, and $70 \%$ for the infraspinatus muscle, with a significant difference between a decrease to $90 \%$ and a decrease to $70 \%(p<0.05)$.

The change over time showed no significant difference for the supraspinatus muscle or the rhomboideus muscle, although showing a significant difference was found for the infraspinatus muscle $-25.0 \pm 8.8$ immediately after exercise and $-3.7 \pm 12.0$ after $72 \mathrm{hr}$ at a decrease to $70 \%(p<0.05$, Figure 1).

The recovery process of each muscle was investigated in the respective conditions based on the standard deviation over $72 \mathrm{hr}$ of daily life, as usual. Results show that all the muscles deviated from and were below the range of $\pm 2 \mathrm{SD}$ at least once at a decrease to $70 \%$, and that they returned to the range of $\pm 2 \mathrm{SD}$ after $72 \mathrm{hr}$ (supraspinatus muscle, Figure 2; infraspinatus muscle, Figure 3). However, the rhomboideus muscle showed kinetics different from the other two muscles, where it returned to the range of \pm 2 SD once but it was below the range again after $72 \mathrm{hr}$ at a decrease to $80 \%$ (Figure 4 ).

\section{Discussion}

The change in muscle hardness and its recovery process were investigated in 7 healthy adult men when internal and external rotations of the shoulder joint were repeated until a predetermined degree of muscle weakness was reached. Results show that a muscle, and particularly the infraspinatus muscle, was stiffer when more muscle weakness existed in the shoulder joint. The supraspinatus muscle and the rhomboideus muscle were also stiffer, exceeding daily changes when there was more muscle weakness in the shoulder joint in internal and external rotations. Results show that the infraspinatus muscle might similarly return to the initial state after $72 \mathrm{hr}$.

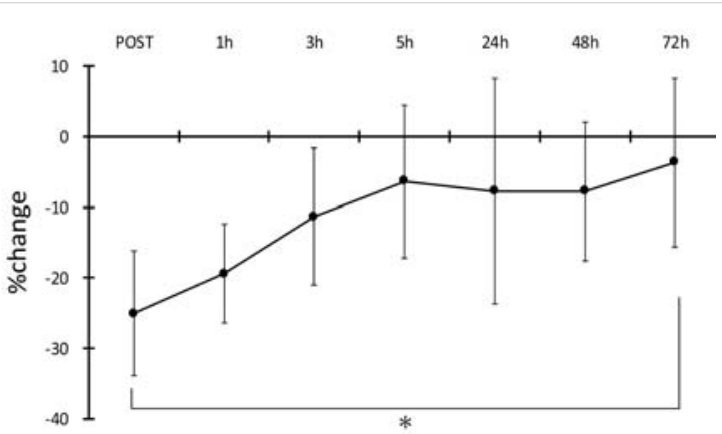

Figure 1: Percent Changes in muscle hardness of the infraspinatus muscle immediately after, and $1,3,5,24,48$ and $72 \mathrm{hr}$ exercise at a decrease to $70 \%$. Date are mean \pm SD. ${ }^{*} p<0.05$ 

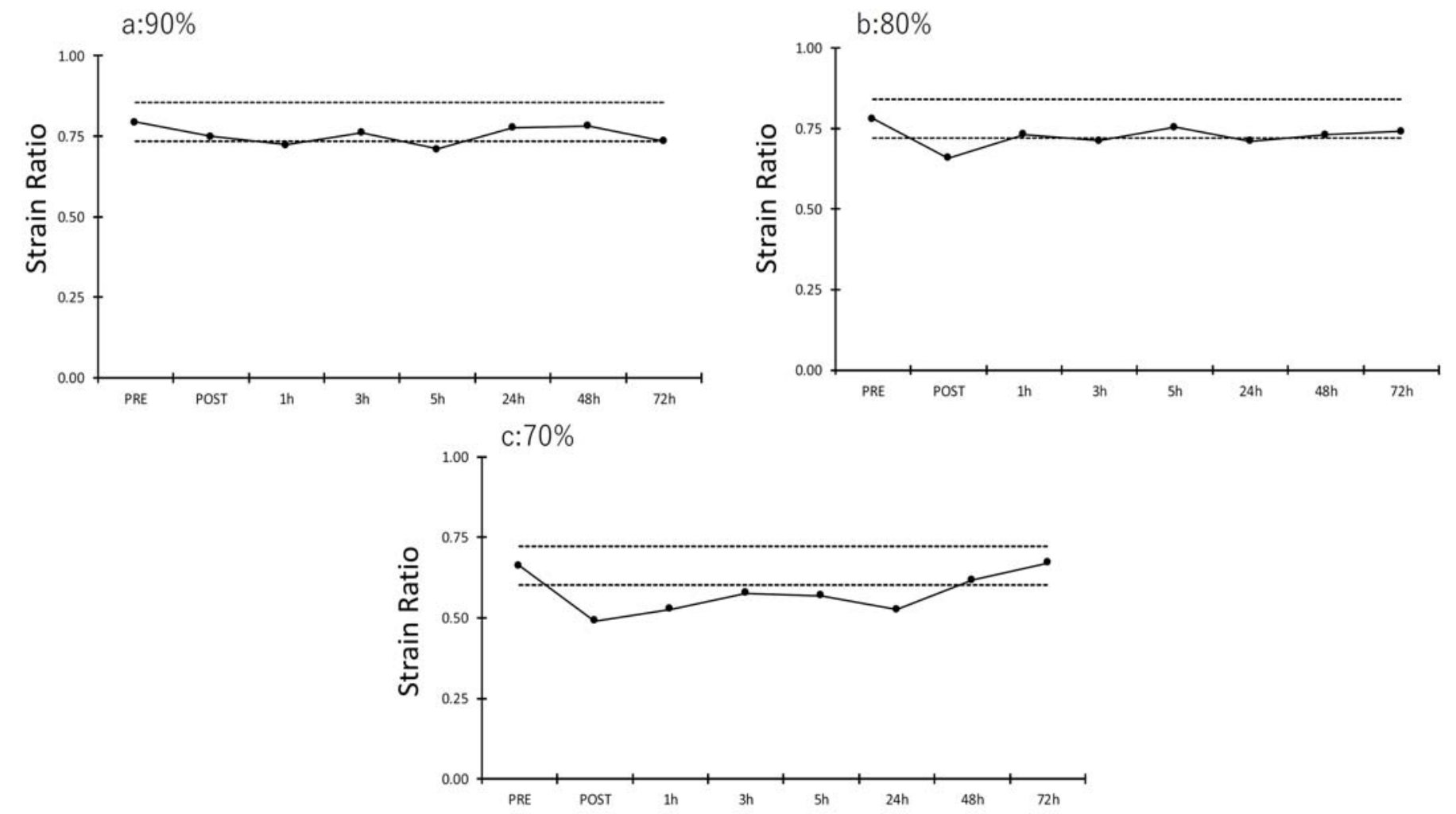

Figure 2: Strain ratio (muscle hardness) of the supraspinatus muscle before and immediately after, at 1, 3, 5, 24, 48 and $72 \mathrm{hr}$ after each exercise bout. Data are mean \pm SD. The dotted line indicates the range of \pm 2 SD. a: degree of muscle weakness is a decrease to $90 \%$. b: degree of muscle weakness is a decrease to $80 \%$. c: degree of muscle weakness is a decrease to $70 \%$.

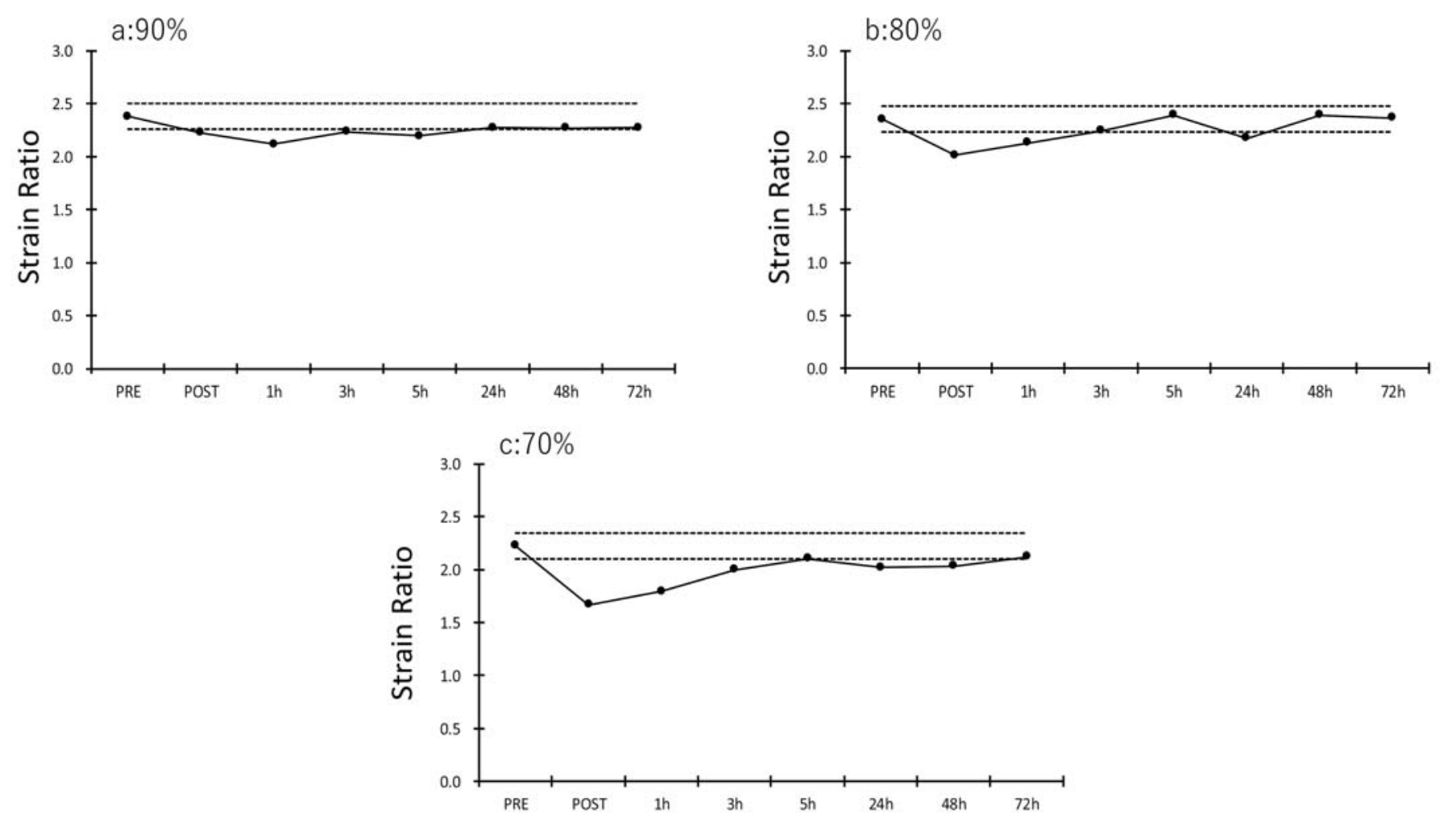

Figure 3: Strain ratio (muscle hardness) of the infraspinatus muscle before and immediately after, at 1, 3, 5, 24, 48 and 72 hr after each exercise bout. Data are mean $\pm S D$. The dotted line indicates the range of \pm 2 SD.

a: degree of muscle weakness is a decrease to $90 \%$. b: degree of muscle weakness is a decrease to $80 \%$. c: degree of muscle weakness is a decrease to $70 \%$. 

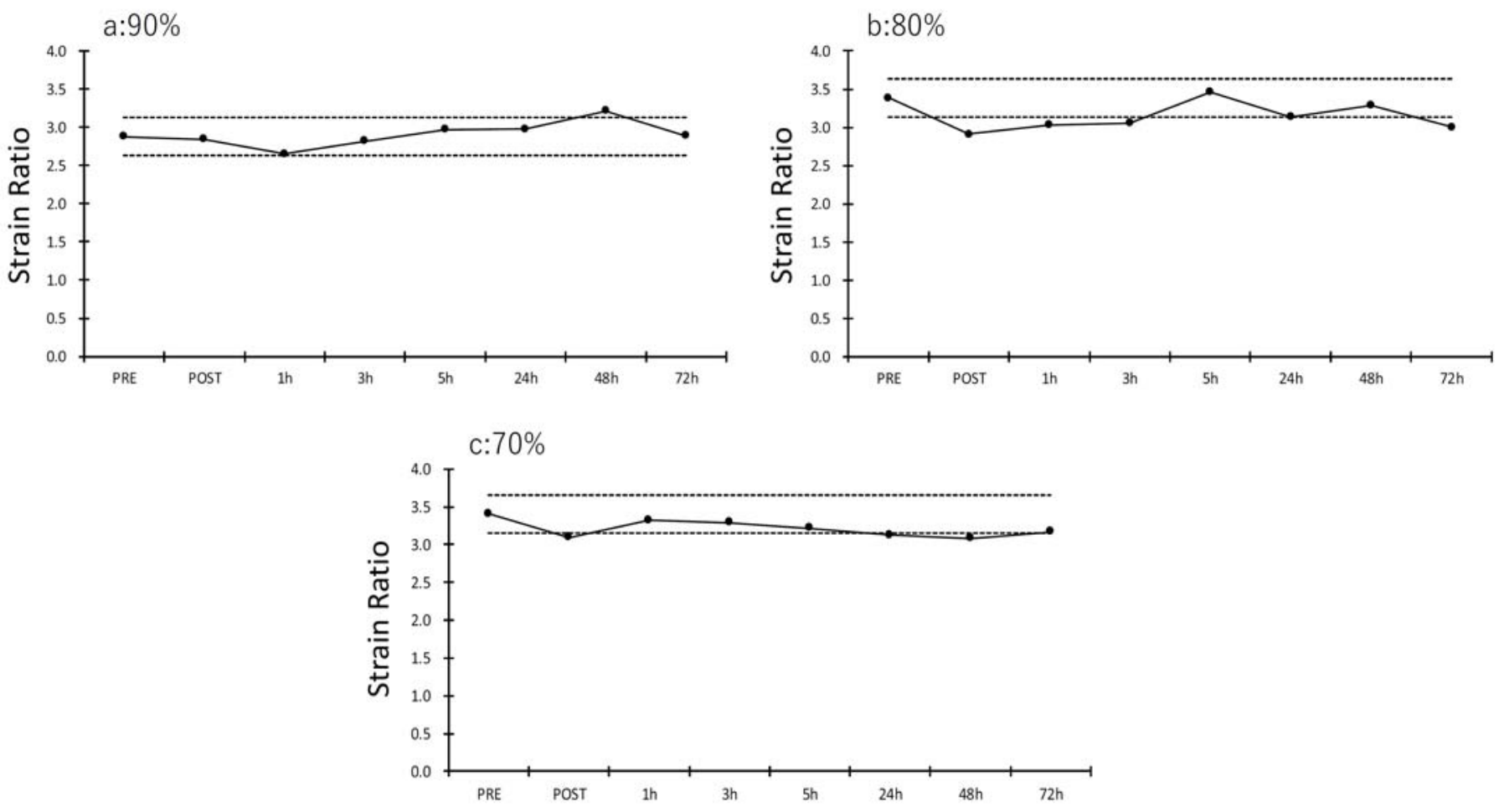

Figure 4: Strain ratio (muscle hardness) of the rhomboideus muscle before and immediately after, at 1, 3, 5, 24, 48 and $72 \mathrm{hr}$ after each exercise bout. Data are mean $\pm \mathrm{SD}$. The dotted line indicates the range of $\pm 2 \mathrm{SD}$.

a: degree of muscle weakness is a decrease to $90 \%$. b: degree of muscle weakness is a decrease to $80 \%$. c: degree of muscle weakness is a decrease to $70 \%$.

Earlier reports have also described that muscles become stiff by exercise [16-18]. Particularly for changes in muscle stiffness and hardness after eccentric exercise, inflammatory reaction associated with muscle damage enhances blood flow and the accumulation of metabolites (fluid accumulation) in and around muscles [19]. It is also considered that an increase in intramuscular pressure caused by these is involved in the change in muscle stiffness immediately after exercise, although it is possible that muscle swelling is involved in the change in muscle stiffness after at least $48 \mathrm{hr}$ [20-22]. In this study, only concentric exercise using the BIODEX device was performed to repeat internal and external rotations in the shoulder joint until clear muscle weakness occurred, taking safety into consideration. Particularly, results demonstrated that the infraspinatus muscle was stiffer immediately after exercise at any hypofunction level. The infraspinatus muscle acts as an agonist in the external rotation of the shoulder joint, and is regarded as more affected by a change in the blood flow because of exercise than the supraspinatus muscle and the rhomboideus muscle, producing changes that are clearer than those of the other two muscles. However, the supraspinatus muscle is a component muscle of the rotator cuff. Also, the rhomboideus muscle plays an important role in maintaining the scapula position during vigorous arm movements. It is considered based on these that the supraspinatus muscle and the rhomboideus muscle also were stiffer and that they deviated from changes that might occur in daily life. For that reason, it is considered that a close relation exists between the degree of hypofunction of the shoulder joint and the conditions of muscles that comprise the rotator cuff and those that surround the scapula: it is presumed that an increase in the strength and amount of pitching can be a direct cause of muscle condition deterioration by increasing load on muscles surrounding the shoulder joint and the scapula.

Actually results show that such changes in muscle hardness might recover to the initial state by resting for $72 \mathrm{hr}$ (3 days). Considering that muscle swelling might be involved in an increase in muscle stiffness, it is likely that icing after pitching is effective to prevent increased muscle stiffness and hardness, although it is necessary to investigate the effects of cooling a muscle itself on changes in muscle stiffness and hardness. Moreover, actual pitching might be associated not only with the concentric contraction performed in this study but also eccentric contraction. Earlier reports have indicated that muscle swelling after $24 \mathrm{hr}$ and more are observed at least in eccentric exercise associated with muscle damage [23-25]. Because the exercises performed in this study consisted only of concentric exercises, induced significant muscle weakness in the shoulder joint was not associated with clear muscle pain or limitation of the range of motion. The rhomboideus muscle showed changes different from the supraspinatus muscle and the infraspinatus muscle. One reason for this is probably that the rhomboideus muscle located around the scapula and the two muscles located around the shoulder joint differ in function during internal and external rotations in the shoulder joint. At least it is likely that the supraspinatus muscle and the infraspinatus muscle, which are also involved in the stability of the shoulder joint requires resting for not 
less than 3 days to recover from increased muscle hardness such as that found in this study to the initial state. However, considering the kinetics of muscle hardness in the rhomboideus muscle, it cannot be denied that other muscles might be stiffer again after $72 \mathrm{hr}$ and more. It is also possible that interventions such as stretching and icing must be done in addition to simple resting for rapid recovery of muscle hardness to the initial state.

\section{Conclusion}

This study demonstrated that the infraspinatus muscle and the supraspinatus muscle become stiffer immediately after exercise when the shoulder joint is at a higher degree of muscle weakness. Results also showed that the change is likely to recover to the initial state after $72 \mathrm{hr}$.

\section{Acknowledgment}

This work was supported by the Joint Research Program of Juntendo University, Faculty of Health and Sports Science.

\section{References}

1. Baltaci G, Johnson R, Kohi $H$ 3rd. Shoulder range of motion characteristics in collegiate baseball players. J Sports Med Phys Fitness. 2001; 41: 236-242.

PubMed: https://www.ncbi.nlm.nih.gov/pubmed/11447368

2. Oliver GD, Weimar WH. Hip and Shoulder Range of Motion in Youth Baseball Pitchers. J Strength Cond Res. 2016; 30: 2823-2827. PubMed: https://www.ncbi.nlm.nih.gov/pubmed/25486297

3. Crockett HC, Gross LB, Wilk KE, Schwartz ML, Reed J, et al. Osseous adaptation and range of motion at the glenohumeral joint in professional baseball pitchers. Am J Sports Med. 2002; 30: 20-26.

PubMed: https://www.ncbi.nlm.nih.gov/pubmed/11798991

4. Amin NH, Ryan J, Fening SD, Soloff L, Schickendantz MS, et al. The Relationship Between Glenohumeral Internal Rotation Deficits, Total Range of Motion, and Shoulder Strength in Professional Baseball Pitchers. J Am Acad Orthop Surg. 2015; 23: 789-796.

PubMed: https://www.ncbi.nlm.nih.gov/pubmed/26609146

5. Laudner KG, Wilson JT, Meister K. Elbow isokinetic strength characteristics among collegiate baseball players. Phys Ther Sport. 2012; 13: 97-100.

PubMed: https://www.ncbi.nlm.nih.gov/pubmed/22498150

6. Myers JB, Laudner KG, Pasquale MR, Bradley JP, Lephart SM Glenohumeral range of motion deficits and posterior shoulder tightness in throwers with pathologic internal impingement. Am J Sports Med. 2006; 34: 385-391.

PubMed: https://www.ncbi.nlm.nih.gov/pubmed/16303877

7. Chou PP, Chou YL, Wang YS, Wang RT, Lin HT. Effects of glenohumeral internal rotation deficit on baseball pitching among pitchers og different ages. J Shoulder Elbow Surg. 2018; 27: 599-605.

PubMed: https://www.ncbi.nlm.nih.gov/pubmed/29241660

8. Tyler TF, Mullaney MJ, Mirabella MR, Nicholas SJ, McHugh MP Risk Factors for Shoulder and Elbow Injuries in High School Baseball Pitchers: The Role of Preseason Strength and Range of Motion. Am J Sports Med. 2014; 42: 1993-1999.

PubMed: https://www.ncbi.nlm.nih.gov/pubmed/24893778

9. Bailey LB, Shanley E, Hawkins R, Beattie PF, Fritz S, et al. Mechanisms of Shoulder Range of Motion Deficits in Asymptomatic Baseball Players. Am J Sports Med. 2015; 43: 2783-2793.

PubMed: https://www.ncbi.nlm.nih.gov/pubmed/26403207
10. Takenaga T, Sugimoto K, Goto H, Nozaki M, Fukuyoshi M, et al. Posterior Shoulder Capsules Are Thicker and Stiffer in the Throwing Shoulders of Healthy College Baseball Players: A Quantitative Assessment Using Shear-Wave Ultrasound Elastography. Am J Sports Med. 2015; 43: 2935-2942.

PubMed: https://www.ncbi.nlm.nih.gov/pubmed/26473012

11. Byram IR, Bushnell BD, Dugger K, Charron K, Harrell FE Jr, et al. Preseason shoulder strength measurements in professional baseball pitchers: identifying players at risk for injury. Am J Sports Med. 2010; 38: 1375-1382.

PubMed: https://www.ncbi.nlm.nih.gov/pubmed/20489215

12. Trakis JE, McHugh MP, Caracciolo PA, Busciacco L, Mullaney M, et al. Muscle strength and range of motion in adolescent pitchers with throwing-related pain: implications for injury prevention. Am J Sports Med. 2008; 36: 2173-2178.

PubMed: https://www.ncbi.nlm.nih.gov/pubmed/18596197

13. Clarsen B, Bahr R, Andersson SH, Munk R, Myklebust G. Reduced glenohumeral rotation, external rotation weakness and scapular dyskinesis are risk factors for shoulder injuries among elite male handball players: a prospective cohort study. Br J Sports Med. 2014; 48: 1327-1333.

PubMed: https://www.ncbi.nlm.nih.gov/pubmed/24948083

14. Moreno-Pérez V, Elvira J, Fernandez-Fernandez J, Vera-Garcia FJ. A comparative study of passive shoulder rotation range of motion, isometric rotation strength and serve speed between elite tennis players with and without history of shoulder pain. Int J Sports Phys Ther. 2018; 13: 39-49.

PubMed: https://www.ncbi.nlm.nih.gov/pubmed/29484240

15. Kusano K, Nishishita S, Nakamura M, Tanaka H, Umehara J, et al. Acute effect and time course of extension and internal rotation stretching of the shoulder on infraspinatus muscle hardness. J Shoulder Elbow Surg. 2017; 26: 1782-1788.

PubMed: https://www.ncbi.nlm.nih.gov/pubmed/28684232

16. Nordez A, Guével A, Casari P, Catheline S, Cornu C. Assessment of muscle hardness changes induced by a submaximal fatiguing isometric contraction. J Electromyogr Kinesiol. 2009; 19: 484-491.

PubMed: https://www.ncbi.nlm.nih.gov/pubmed/18158253

17. Yanagisawa $O$, Niitsu $M$, Kurihara $T$, Fukubayashi $T$. Evaluation of human muscle hardness after dynamic exercise with ultrasound real-time tissue elastography: a feasibility study. Clin Radiol. 2011; 66: 815-819. PubMed: https://www.ncbi.nlm.nih.gov/pubmed/21529793

18. Kishimoto K, Sakuraba K, Kubota A, Fujita S. The effect of concentric and eccentric exercise on muscle hardness. Juntendo Med J. 2018; 64: 371-378.

19. Murayama M, Nosaka K, Yoneda T, Minamitani K. Changes in hardness of the human elbow flexor muscles after eccentric exercise. Eur J Appl Physiol. 2000; 82: 361-367.

PubMed: https://www.ncbi.nlm.nih.gov/pubmed/10985588

20. Howell JN, Chleboun G, Conatser R. Muscle stiffness, strength loss, swelling and soreness following exercise-induced injury in humans. $J$ Physiol. 1993; 464: 183-196.

PubMed: https://www.ncbi.nlm.nih.gov/pubmed/8229798

21. Green MA, Sinkus R, Gandevia SC, Herbert RD, Bilston LE. Measuring changes in muscle stiffness after eccentric exercise using elastography. NMR Biomed. 2012; 25: 852-858.

PubMed: https://www.ncbi.nlm.nih.gov/pubmed/22246866

22. Lacourpaille L, Nordez A, Hug F, Couturier A, Dibie C, et al. Timecourse effect of exercise-induced muscle damage on localized muscle mechanical properties assessed using elastography. Acta Physiol (Oxf). 2014; 211: 135-146.

PubMed: https://www.ncbi.nlm.nih.gov/pubmed/24602146 
23. Proske U, Allen TJ. Damage to skeletal muscle from eccentric exercise. Exerc Sport Sci Rev. 2005; 33: 98-104.

PubMed: https://www.ncbi.nlm.nih.gov/pubmed/15821431

24. Peake J, Nosaka K, Suzuki K. Characterization of inflammatory responses to eccentric exercise in humans. Exerc Immunol Rev. 2005;
11: 64-85.

PubMed: https://www.ncbi.nlm.nih.gov/pubmed/16385845

25. Peake JM, Neubauer O, Della Gatta PA, Nosaka K. Muscle damage and inflammation during recovery from exercise. J Appl Physiol. 2017; 122: 559-570.

PubMed: https://www.ncbi.nlm.nih.gov/pubmed/28035017 